\title{
Population Pharmacokinetics of Cefazolin in Serum and Tissue for Patients with Complicated Skin and Soft Tissue Infections (cSSTI)
}

\author{
Wonhee So · Joseph L. Kuti · David P. Nicolau
}

To view enhanced content go to www.infectiousdiseases-open.com

Received: October 4, 2014 / Published online: November 20, 2014

(C) The Author(s) 2014. This article is published with open access at Springerlink.com

\section{ABSTRACT}

Introduction: Cefazolin is commonly used to treat complicated skin and soft tissue infections (cSSTI) caused by methicillin-susceptible Staphylococcus aureus (MSSA) and Enterobacteriaceae. We aimed to determine the variability of cefazolin exposure in interstitial fluid (ISF) of tissue and evaluate its dosing recommendations.

Methods: Population pharmacokinetics were performed to co-model serum and ISF concentration data from six patients enrolled in a previous in vivo microdialysis study. A 5,000 patient Monte Carlo simulation was then conducted for 1 and 2 g every $8 \mathrm{~h}$ (q8h) regimens to calculate the penetration ratio and probability of target attainment (PTA) at 30\% and $50 \%$ of the

Electronic supplementary material The online version of this article (doi:10.1007/s40121-014-0049-3) contains supplementary material, which is available to authorized users.

W. So · J. L. Kuti · D. P. Nicolau $(\bowtie)$

Center for Anti-Infective Research and

Development, Hartford Hospital, Hartford, CT, USA

e-mail: david.nicolau@hhchealth.org

D. P. Nicolau

Division of Infectious Diseases, Hartford Hospital,

Hartford, CT, USA dosing interval that free drug concentrations remain above the minimum inhibitory concentration ( $f \mathrm{~T}>\mathrm{MIC}$ ) in ISF of tissue.

Results: A three-compartment model, with one of the compartments representing ISF concentrations, fits the data best. The final model resulted in the mean $\pm S D$ parameter values: Clearance $=3.8 \pm 2.1 \mathrm{~L} / \mathrm{h}$, volume of distribution in central compartment $=8.6 \pm$ $6.4 \mathrm{~L}$ and volume of distribution in ISF $=$ $36.6 \pm 17.9 \mathrm{~L}$. The mean $\pm \mathrm{SD}$ and median penetration ratios were $1.36 \pm 4.57$ and 0.80 , respectively. At the $\mathrm{MIC}_{90}$ for MSSA of $1 \mathrm{mg} / \mathrm{L}$, PTAs for the $1 \mathrm{~g}$ q8h dose in ISF were 96\% and 91\% for $30 \%$ and $50 \% f \mathrm{~T}>$ MIC targets, respectively, which decreased to $87 \%$ and $71 \%$ at $2 \mathrm{mg} / \mathrm{L}$. For the same respective targets, a $2 \mathrm{~g}$ q8h dosing regimen increased PTA to $96 \%$ and $91 \%$ at $2 \mathrm{mg} / \mathrm{L}$. Conclusion: Cefazolin penetration into the ISF of a lower limb infection varied across this simulated patient population. Based on these data, a $1 \mathrm{~g}$ q8h regimen should be sufficient to obtain 30\% $f \mathrm{~T}>\mathrm{MIC}$ exposure against most MSSA causing cSSTI. However, a $2 \mathrm{~g}$ q8h dose is required to obtain $50 \% \quad f \mathrm{~T}>\mathrm{MIC}$ pharmacodynamic targets at the current breakpoint for Enterobacteriaceae $(2 \mathrm{mg} / \mathrm{L})$. 
Keywords: Cefazolin; cSSTI; Diabetic wound; Microdialysis; Tissue pharmacokinetics

\section{INTRODUCTION}

Methicillin-susceptible Staphylococcus aureus (MSSA) is one of the most common causes of complicated skin and soft tissue infection (cSSTI) [1]. Among deeper cSSTI and those located in the lower limb of diabetic patients, Gram-negative bacilli, Escherichia coli, Klebsiella pneumoniae, and Proteus species, are also commonly isolated $[1,2]$. Based on this epidemiology, intravenous cefazolin has often been used to treat cSSTI for organisms proven susceptible in the hospital settings. However, dosing recommendations vary based on source and organism. Current US labeling for cefazolin includes a variety of dosing regimens as low as $250 \mathrm{mg}$ every $8 \mathrm{~h}$ (q8h) up to $2 \mathrm{~g}$ q8h [3].

Susceptibility breakpoints also vary for cefazolin; the U.S. Food and Drug Administration (FDA), the European Committee on Antimicrobial Susceptibility Testing (EUCAST), or the Clinical Laboratory Standards Institute (CLSI) breakpoints have not taken cefazolin tissue exposure into account when selecting susceptibility thresholds. The FDA susceptibility breakpoint for cefazolin against $S$. aureus has remained $\leq 16 \mathrm{mg} / \mathrm{L}$ for decades [3], while cefazolin minimum concentration at which 50\% $\left(\mathrm{MIC}_{50}\right)$ and $90 \%$ $\left(\mathrm{MIC}_{90}\right)$ of the isolates were inhibited against MSSA was recently reported as $0.5 \mathrm{mg} / \mathrm{L}$ and $1 \mathrm{mg} / \mathrm{L}$, respectively [4]. EUCAST has not published a cefazolin susceptibility breakpoint for Enterobacteriaceae and relies on the cefoxitin disc test for determining universal susceptibility to first-generation cephalosporins. The CLSI uses a similar definition for $S$. aureus, and more recently has modified its susceptibility breakpoint for Enterobacteriaceae twice since 2010. This was prompted by publications reporting treatment failures from cephalosporins in infections caused by Enterobacteriaceae that were previously categorized as susceptible [5-7]. Initially, it was lowered from $\leq 8 \mathrm{mg} / \mathrm{L}$ to $\leq 1 \mathrm{mg} / \mathrm{L}$ in 2010 with the advent of in vitro susceptibility, pharmacodynamic, and clinical outcome data. It was then readjusted to $\leq 2 \mathrm{mg}$ / $\mathrm{L}$ in 2011 with the recommendation of a $2 \mathrm{~g}$ q8h dosing regimen, so that it could still be a viable option for Enterobacteriaceae without intrinsic chromosomal cephalosporinases [7].

In an effort to quantify drug concentrations in the interstitial fluid (ISF) of tissue to determine if appropriate pharmacodynamic targets are achieved at the site of infection, our group previously reported ISF exposure of cefazolin in seven chronic lower extremity wound infections utilizing in vivo microdialysis techniques $[8,9]$. The noncompartmental pharmacokinetic analysis from the study showed the mean and median tissue penetration ratios [ISF/serum free drug area under the curve (fAUC)] of 1.06 and 0.88 with a wide range of $0.19-1.68$. The percent of the dosing interval that free drug concentrations remained above the MIC (\% $\mathrm{T}>\mathrm{MIC})$ in the sampled ISF was $100 \%$ at an MIC of $1 \mathrm{mg} / \mathrm{L}$ for five out of six patients who received $1 \mathrm{~g}$ q8h dose during the study.

In this study, we aimed to describe the population pharmacokinetics of cefazolin in both serum and ISF utilizing the data from the aforementioned study [8]. We used this model to simulate the potential variability in penetration into ISF of tissue and to compare the likelihood of achieving targeted drug exposure (i.e., $f \mathrm{~T}>\mathrm{MIC}$ ) in the ISF compartment between $1 \mathrm{~g}$ versus $2 \mathrm{~g} \mathrm{q} 8 \mathrm{~h}$ regimens. 


\section{METHODS}

\section{Patient Population and Setting}

Included patients were those from the aforementioned in vivo microdialysis study, which was an open-label pharmacokinetic study at Hartford Hospital, Hartford, CT, USA [8]. Inclusion criteria were hospitalized adult patients (age $\geq 18$ years) with lower extremity wound infections requiring surgical debridement and defined as mild or moderate by the Infectious Diseases Society of America or as grade 2 or 3 by the International Consensus on the Diabetic Foot [10]. Patients with hypersensitivity to anesthetics (lidocaine or lidocaine derivatives), pregnancy or breastfeeding, no palpable pedal pulses, likelihood to require multiple procedures during the study, participation in another study of an investigational drug or device within the preceding 30 days, and patients with renal dysfunction, defined as an estimated creatinine clearance $(\mathrm{CrCl})$ less than $50 \mathrm{~mL} / \mathrm{min}$, were excluded. The analysis in this article is based on a previously conducted study and does not involve any new studies with human or animal subjects performed by any of the authors.

\section{Drug Administration}

All patients received intravenous cefazolin over 30 or $60 \mathrm{~min}$ for their lower extremity wound infections via a peripheral catheter placed in the arm or a peripherally inserted central catheter (PICC). All patients included in this analysis received $1 \mathrm{~g}$ q8h dosing.

\section{Sampling and Determination of Cefazolin Concentrations}

Venous blood samples (7-9 per patient) were collected at various time points from a peripheral intravenous catheter or PICC after a minimum of four doses (i.e., steady state). Sampling time points included immediately prior to and after the infusion, several points hourly after the infusion, and then just prior to the next dose. Dialysate samples of $\sim 120 \mu \mathrm{L}$ were obtained from the microdialysis catheters at each of the corresponding blood sample time points. Microdialysis catheters were calibrated for each patient after sampling by using the in vivo retrodialysis technique [11]. Cefazolin concentrations in serum and ISF were quantified by a validated high-performance liquid chromatography assay [12].

\section{Population Pharmacokinetics}

Cefazolin serum and ISF concentrations were co-modeled by the non-parametric adaptive grid (NPAG) with adaptive gamma algorithm available in the Pmetrics package for R (LAPK, Los Angeles, CA, USA) [13]. A total of 53 serum and 53 ISF concentrations from six patients were used, and individual concentrations were weighted by the reciprocal of assay variance multiplied by gamma. Weighting based on interday assay variance was employed using a plot of the assay standard deviation (SD) versus measured cefazolin concentrations, which was best described by the equations: $\mathrm{SD}_{\text {serum }}=$ $\gamma(0.0071+0.0449 \times C 1)$ and $\mathrm{SD}_{\mathrm{ISF}}=\gamma(0.0002$ $+0.0560 \times C 2$ ), where $C 1$ and $C 2$ are cefazolin concentrations in serum and ISF, respectively, and $\gamma$ was identified as 3.76. Mean values were used as the measure of central tendency for population parameter estimates. Bayesian estimates were obtained for each patient using the population-of-one utility within Pmetrics.

Both two- and three-compartment models were explored, with one of the compartments being the sampled ISF. Multiple models were evaluated and discriminated employing the 
Akaike Information Criterion (AIC) [14], which is a function of the likelihood of the model, penalized by the number of parameters in the model, bias, imprecision, and by visual inspection of the observed versus predictive concentration plots. The mean weighted error was used as the estimate of bias. The biasadjusted mean weighted squared error was employed as the estimate of imprecision.

Linear regression (Sigma Plot Version 12.5, Systat, Chicago, IL, USA) was used to characterize the relationship between pharmacokinetic parameters (CL, clearance from central compartment; $V_{\mathrm{c}}$, volume of central compartment and $V_{\mathrm{ISF}}$, volume of sampled ISF compartment) and patient covariates ( $\mathrm{CrCl}$ calculated by Cockcroft-Gault equation $[15,16]$, TBW, total body weight and BMI, body mass index). Covariates that were statistically significantly correlated were then incorporated back into the population model and tested for model superiority.

\section{Monte Carlo Simulations}

A 5,000 patient semi-parametric Monte Carlo Simulation (Pmetrics) [13] was conducted to simulate steady-state concentrations of cefazolin $1 \mathrm{~g}$ and $2 \mathrm{~g}$ in both serum and ISF. Pmetrics' semi-parametric simulator uses the non-parametric "support points" from the final population model, each a vector of one value for each parameter in the model and the associated probability of that set of parameter values, to serve as the mean of one multivariate normal distribution. The weight of each multivariate distribution is equal to the probability of the point. The overall population covariance matrix is divided by the number of support points and applied to each distribution for sampling. Cefazolin concentrations in serum and tissue for seven doses of each regimen were simulated every 15 min after the last dose. A fraction unbound of $15 \%$ (i.e., $85 \%$ protein binding) was applied to correct all serum concentrations to free drug concentrations. This fraction unbound estimate was consistent across the six patients, as well as within each patient across the dosing interval [8]. ISF concentrations were assumed to be unbound during calculation of AUC and $f \mathrm{~T}>\mathrm{MIC}$ in ISF. The AUC for a dosing interval of $8 \mathrm{~h}\left(\mathrm{AUC}_{0-8}\right)$ at steady state was estimated in serum and ISF after simulation to calculate the penetration into ISF as $\mathrm{AUC}_{\mathrm{ISF}} / \mathrm{fAUC}$ serum. The probability of target attainment (PTA) was calculated using a priori pharmacodynamic targets defined as $30 \%, 50 \%$, and $100 \%$ $f \mathrm{~T}>\mathrm{MIC}$ in serum and ISF.

\section{RESULTS}

\section{Patient Characteristics}

A total of six patients were included in this analysis. Five out of six patients were male and their age ranged from 35 to 83 years with a mean \pm SD of $55 \pm 17$ years (Table 1 ). Five out of six patients had diabetes (one with type I, four patients with type II). One patient did not have diabetes but had significant neuropathy, which contributed to the foot infection. None of the six patients had a history of peripheral vascular disease, and all had pedal pulses from +1 to +3 at the time of sampling. Surgical interventions involved were amputation or debridement of the infected tissue and/or bone. Sampling occurred after the surgical intervention for five out of the six patients except in one patient, which occurred a day prior to surgery. 


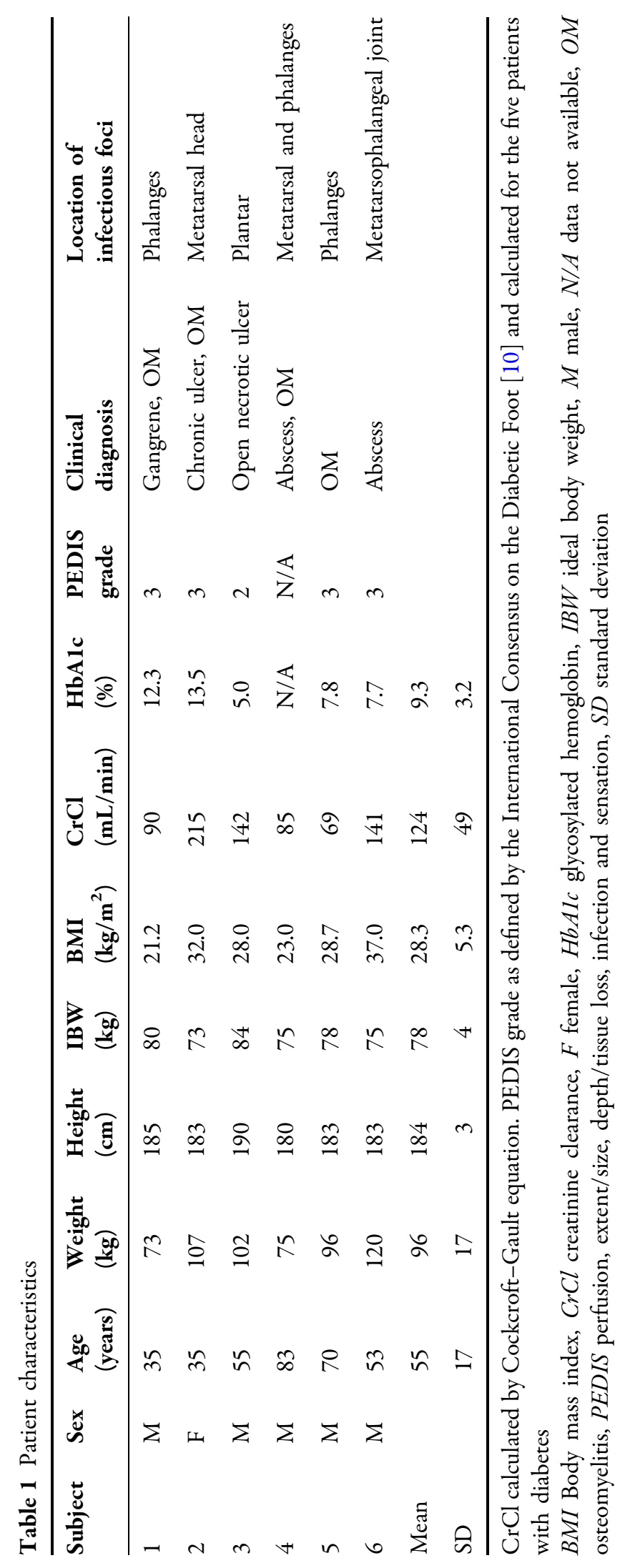




\section{Population Pharmacokinetics}

A three-compartment base model fit the data better than a two-compartment model based on our discrimination criteria. Although linear regression identified $\mathrm{CrCl}$ as the only patient covariate that was significantly correlated with CL $\left(r^{2}=0.822, \quad P=0.005\right)$ and no patient covariates were significantly correlated with $V_{\mathrm{c}}$ or $V_{\text {ISF }}$, incorporation of $\mathrm{CrCl}$ back into the base model did not improve the AIC. Therefore, the simpler non-covariate three-compartment model was selected. The final model AIC and log-likelihood were 453.3 and -218.8, respectively. Final population pharmacokinetic parameters obtained from the analysis are listed in Table 2.

Figure 1 demonstrates the observed versus (Fig. 1a) population predicted and (Fig. 1b) individual predicted maximum posterior (MAP) concentrations in serum. The population predicted estimates were reasonable for a patient population with variable pharmacokinetics with $r^{2}$, bias, and imprecision values of $0.64,12.7$, and 920, respectively (Fig. 1a). MAP Bayesian concentration plot in serum was excellent with $r^{2}$, bias, imprecision values of 0.98 , -0.373 , and 7.6 , respectively (Fig. $1 b$ ). In ISF, the observed versus population predicted $r^{2}$, bias, and imprecision were 0.198, 20.1, and 1,394 (Fig. 2a), and the corresponding values for the observed versus individual MAP Bayesian plot were $0.887,-1.17$, and 21.3 , respectively (Fig. 2b).

The mean $\pm \mathrm{SD}$ and median penetration ratios for the six included patients based on their individual Bayesian parameter estimates were $0.90 \pm 0.48$ and 0.72 , respectively.

\section{Monte Carlo Simulation}

Parameter estimates from the Pmetrics semiparametric simulator recapitulated the mean, SD, and median estimates of the original model. The mean \pm SD and median penetration ratios for 5,000 simulated patients were $1.36 \pm 4.57$ and 0.80, respectively. The 5th and 95th percentiles for simulated penetration into ISF were 0.15 and 2.74 , respectively. PTA results for serum and ISF exposures of $30 \%, 50 \%$, and $100 \% f \mathrm{~T}>$ MIC are provided in Table 3.

\section{DISCUSSION}

The previously published cefazolin in vivo microdialysis study by our group evaluated ISF concentrations of intravenously administered

Table 2 Final parameter estimates from the population pharmacokinetic model

\begin{tabular}{lccccccc}
\hline Parameter & $\mathbf{C L}(\mathbf{L} / \mathbf{h})$ & $\boldsymbol{V}_{\mathbf{c}}(\mathbf{L})$ & $\boldsymbol{K}_{\mathbf{1 2}}\left(\mathbf{h}^{-\mathbf{1}}\right)$ & $\boldsymbol{K}_{\mathbf{2 1}}\left(\mathbf{h}^{-\mathbf{1}}\right)$ & $\boldsymbol{K}_{\mathbf{1 3}}\left(\mathbf{h}^{-\mathbf{1}}\right)$ & $\boldsymbol{K}_{\mathbf{3 1}}\left(\mathbf{h}^{-\mathbf{1}}\right)$ & $\boldsymbol{V}_{\mathbf{I S F}}(\mathbf{L})$ \\
\hline Mean & 3.8 & 8.6 & 1.6 & 4.0 & 1.4 & 2.0 & 36.6 \\
$\mathrm{SD}$ & 2.1 & 6.4 & 1.6 & 1.6 & 1.3 & 1.5 & 17.9 \\
$\% \mathrm{CV}$ & 55.2 & 74.6 & 103.7 & 40.9 & 94.2 & 72.2 & 48.8 \\
Median & 2.8 & 6.5 & 2.1 & 5.0 & 0.9 & 0.9 & 41.0 \\
\hline
\end{tabular}

$C L$ Clearance from central compartment, $K_{12}$ transfer rate constant from central to peripheral compartment, $K_{13}$ transfer rate constant from central to the sampled interstitial fluid of tissue compartment, $K_{21}$ transfer rate constant from peripheral to central compartments, $K_{31}$ transfer rate constant from the sampled interstitial fluid of tissue compartment to central compartment, $S D$ standard deviation, $\% C V$ coefficient of variation, $V_{c}$ volume of the central compartment, $V_{I S F}$ volume of the sampled interstitial fluid of tissue compartment 

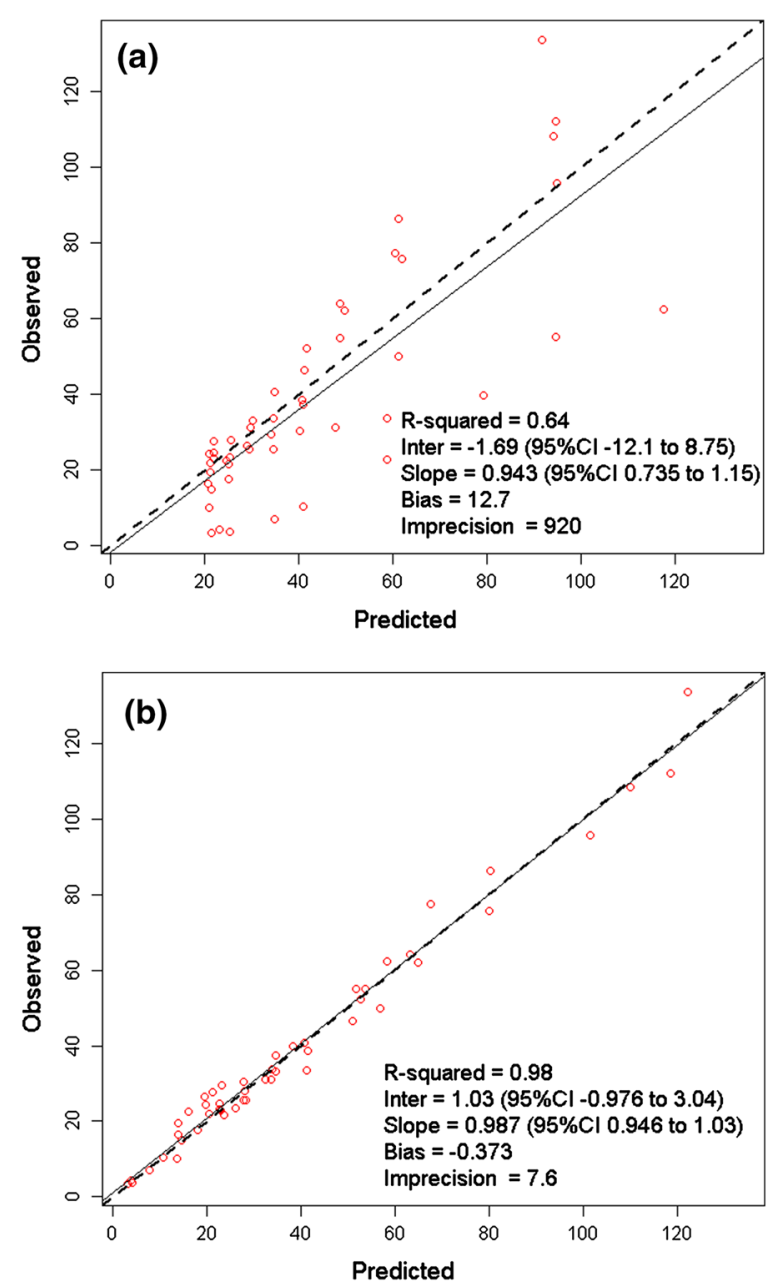

Fig. 1 Observed versus (a) population predicted and (b) individual predicted maximum posterior Bayesian cefazolin concentrations $(\mathrm{mg} / \mathrm{L})$ in serum. CI Confidence interval, Inter Intercept

cefazolin for the treatment of cSSTI [8]. The primary goal of the current study was to describe the population pharmacokinetics using the same serum and ISF concentration data to assess variability in ISF penetration and the likelihood of achieving $f \mathrm{~T}>\mathrm{MIC}$ in the ISF compartment between $1 \mathrm{~g}$ and $2 \mathrm{~g}$ q8h regimens.

A three-compartment model best described the pharmacokinetics of cefazolin in our population with normal renal function. The resultant $\mathrm{CL}$ of $3.78 \pm 2.09 \mathrm{~L} / \mathrm{h}$ from our
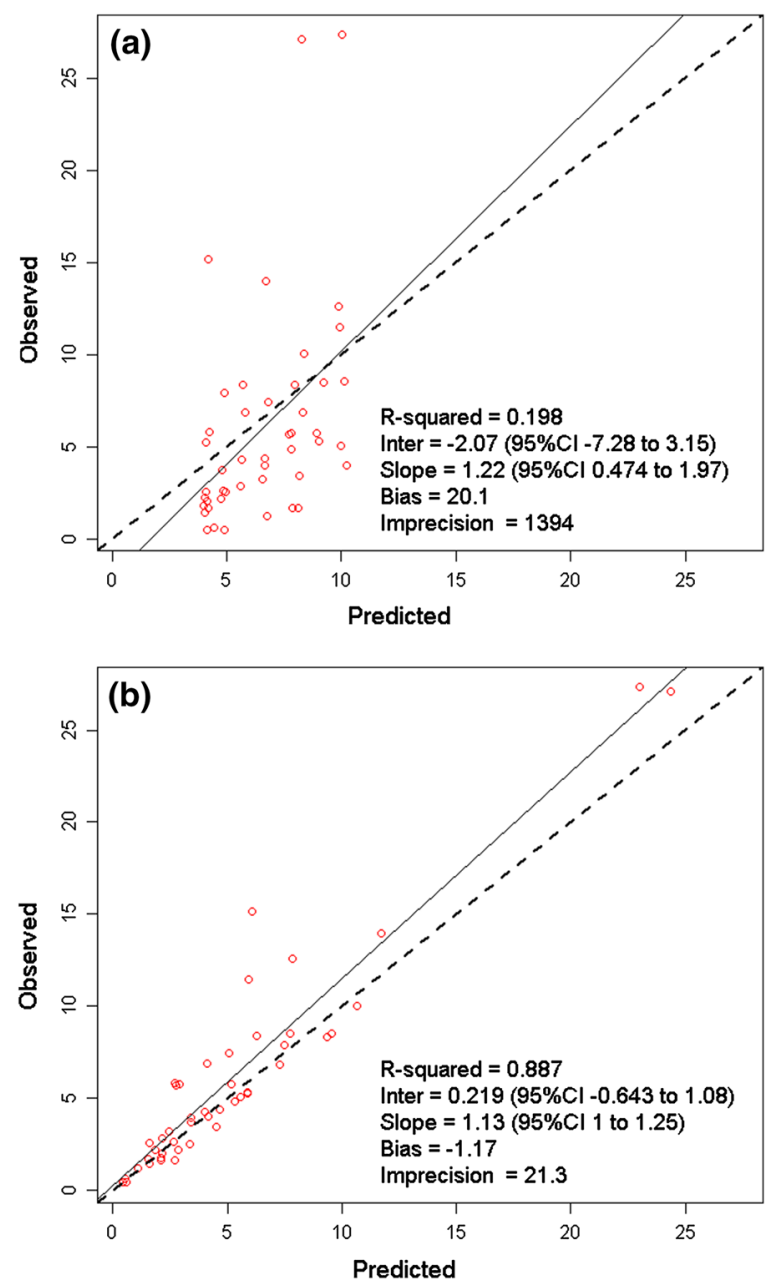

Fig. 2 Observed versus (a) population predicted and (b) individual predicted maximum posterior Bayesian cefazolin concentrations $(\mathrm{mg} / \mathrm{L})$ in interstitial fluid of tissue. CI Confidence interval, Inter Intercept

population pharmacokinetic model was concordant with the values from the noncompartmental analysis, $3.72 \pm 2.16 \mathrm{~L} / \mathrm{h}$ [8]. Cefazolin $\mathrm{CL}$ in this population was also similar to most other reported values. van Kralingen and colleagues [17] have reported $\mathrm{CL}$ of $4.2 \pm 0.1 \mathrm{~L} / \mathrm{h}$ in patients with average \pm SD age of $44 \pm 11$ years, TBW of $151 \pm 35 \mathrm{~kg}$, and BMI of $51 \pm 10 \mathrm{~kg} / \mathrm{m}^{2}$ after a single prophylactic dose of $2 \mathrm{~g}$ prior to bariatric surgery. They also observed CL had a significant negative correlation with age, but not with 
Table 3 Probability of achieving pharmacodynamic exposure thresholds in serum (a) and interstitial fluid of tissue (b) for cefazolin $1 \mathrm{~g}$ and $2 \mathrm{~g}$ q8h dosing regimens from 5,000 patient Monte Carlo simulation

\begin{tabular}{|c|c|c|c|c|c|c|}
\hline \multirow[t]{2}{*}{$\mathrm{MIC}(\mathrm{mg} / \mathrm{L})$} & \multicolumn{3}{|l|}{$1 \mathrm{~g} \mathrm{q} 8 \mathrm{~h}$} & \multicolumn{3}{|l|}{$2 \mathrm{~g} \mathrm{q} 8 \mathrm{~h}$} \\
\hline & $30 \% f \Gamma>M I C$ & $50 \% f \mathrm{~T}>\mathrm{MIC}$ & $100 \% f \mathrm{~T}>\mathrm{MIC}$ & $30 \% f \Gamma>M I C$ & $50 \% f \Gamma>M I C$ & $100 \% f \mathrm{~T}>\mathrm{MIC}$ \\
\hline \multicolumn{7}{|l|}{ (a) Serum } \\
\hline 0.125 & 1.00 & 0.99 & 0.96 & 1.00 & 0.99 & 0.96 \\
\hline 0.25 & 0.99 & 0.99 & 0.93 & 1.00 & 0.99 & 0.96 \\
\hline 0.5 & 0.99 & 0.98 & 0.84 & 0.99 & 0.99 & 0.93 \\
\hline 1 & 0.99 & 0.97 & 0.66 & 0.99 & 0.98 & 0.84 \\
\hline 2 & 0.98 & 0.79 & 0.41 & 0.99 & 0.97 & 0.66 \\
\hline 4 & 0.77 & 0.61 & 0.08 & 0.98 & 0.79 & 0.41 \\
\hline 8 & 0.33 & 0.09 & 0.01 & 0.77 & 0.61 & 0.08 \\
\hline 16 & 0.03 & 0.01 & 0.00 & 0.34 & 0.09 & 0.01 \\
\hline \multicolumn{7}{|c|}{ (b) Interstitial fluid of tissue } \\
\hline 0.125 & 0.99 & 0.98 & 0.93 & 0.99 & 0.99 & 0.95 \\
\hline 0.25 & 0.99 & 0.97 & 0.89 & 0.99 & 0.98 & 0.93 \\
\hline 0.5 & 0.98 & 0.96 & 0.77 & 0.99 & 0.97 & 0.89 \\
\hline 1 & 0.96 & 0.91 & 0.58 & 0.98 & 0.96 & 0.77 \\
\hline 2 & 0.87 & 0.71 & 0.39 & 0.96 & 0.91 & 0.58 \\
\hline 4 & 0.58 & 0.45 & 0.20 & 0.87 & 0.71 & 0.39 \\
\hline 8 & 0.29 & 0.18 & 0.08 & 0.58 & 0.45 & 0.20 \\
\hline 16 & 0.10 & 0.07 & 0.03 & 0.29 & 0.18 & 0.08 \\
\hline
\end{tabular}

$f T>M I C$ Free time above MIC, $q 8 h$ every $8 \mathrm{~h}$

body weight. Douglas and colleagues [18] reported median $\mathrm{CL}$ of $3.01 \mathrm{~L} / \mathrm{h}$ (interquartile range: $1.73-3.94 \mathrm{~L} / \mathrm{h}$ ) after a single $2 \mathrm{~g}$ cefazolin in patients undergoing abdominal aortic aneurysm open repair surgery. A tissue penetration study of cefazolin in morbidly obese versus non-obese patients reported a CL of approximately $23 \mathrm{~L} / \mathrm{h}$ [19]; however, this estimate was based on unbound cefazolin concentrations and is therefore not directly comparable. Nonetheless, this unbound CL estimate is feasible because cefazolin is highly protein bound; protein binding in our six patients was $85 \%$.
The observed mean \pm SD and median penetration ratios from our six patients using their individual Bayesian parameter estimates were $0.90 \pm 0.48$ and 0.72 (range 0.61-1.87), respectively; these values were similar to the original observed penetration ratios based on trapezoidal rule: $1.21 \pm 0.67$ and 0.90 (range 0.7-2.68), respectively [8]. The Monte Carlo simulation incorporates variability between patients in the pharmacokinetic estimates, and as a result, the mean $\pm \mathrm{SD}$ and median simulated penetration ratios were $1.36 \pm 4.57$ and 0.80 , respectively. These penetration ratio are comparable with the values observed by Brill 
and colleagues [19] who compared population pharmacokinetics of surgical prophylactic dose of cefazolin $2 \mathrm{~g}$ in both morbidly obese (BMI $47 \pm 6 \mathrm{~kg} / \mathrm{m}^{2}$ ) and non-obese (BMI $28 \pm$ $3 \mathrm{~kg} / \mathrm{m}^{2}$ ) patients using in vivo microdialysis. Their observed $\mathrm{fAUC}_{\mathrm{ISF}} / \mathrm{AUC}_{\text {serum }}$ was 0.70 (range 0.68-0.83) in morbidly obese and 1.02 (range 0.85-1.41) in non-obese patients. The aforementioned study by Douglas and colleagues [18] reported $85 \%$ penetration (range 78-106\%). Taken collectively, these data consistently suggest that there is the potential for a wide range in the estimate for cefazolin penetration into ISF, which may unpredictably be greater than or less than exposures in serum.

While penetration ratio was calculated to display the relative exposure of cefazolin in ISF of tissue versus in serum, the pharmacodynamic target of interest for the efficacy of cefazolin in ISF remains $f \mathrm{~T}>\mathrm{MIC}$ [20-22]. Although the cefazolin target $f T>$ MIC in ISF needed for efficacy against MSSA and Enterobacteriaceae are unknown, 30\% for MSSA [22-24] and 50\% for Enterobacteriaceae $[22,25,26]$ were employed as these are targets required in blood. A target of $100 \% f \mathrm{~T}>\mathrm{MIC}$ was also included for comparison. Consistent with current breakpoints, where applicable, PTAs for cefazolin $1 \mathrm{~g}$ q8h in serum were high at MICs of $1 \mathrm{mg} / \mathrm{L}$ and $2 \mathrm{mg} / \mathrm{L}$ (Table $3 \mathrm{a}$ ). However, analysis of exposure probabilities in tissue revealed much lower PTA results. At the $\mathrm{MIC}_{90}$ for MSSA of $1 \mathrm{mg} / \mathrm{L}$, the PTA for a $1 \mathrm{~g} \mathrm{q} 8 \mathrm{~h}$ dose in ISF was $96 \%$ using the 30\% $f \mathrm{~T}>\mathrm{MIC}$ target (Table 3b). At an MIC of $2 \mathrm{mg} / \mathrm{L}$, the current-susceptible breakpoint for Enterobacteriaceae, a $1 \mathrm{~g} \quad \mathrm{q} 8 \mathrm{~h}$ regimen obtained a PTA of $71 \%$ for $50 \% f \mathrm{~T}>\mathrm{MIC}$ (Table $3 \mathrm{~b}$ ), but a $2 \mathrm{~g}$ q8h dosing regimen increased the PTA to $91 \%$ for the same target (Table 3b). PTAs for 50\% $f \mathrm{~T}>\mathrm{MIC}$ in tissue from our study for the $2 \mathrm{~g}$ dose are comparable with PTAs reported by Brill and colleagues [19]: 96\% and $91 \%$ (Table $3 \mathrm{~b}$ ) versus $100 \%$ and $96 \%$ at MIC of $1 \mathrm{mg} / \mathrm{L}$ and $2 \mathrm{mg} / \mathrm{L}$, respectively.

The primary limitation of our study is its small sample size. Only six patients, all with normal renal function, were included in the final model. Despite the knowledge of correlation between cefazolin CL and renal dysfunction, the small number of patients with normal renal function in our study made it challenging to improve the model by adding this covariate. Additionally, while morbid obesity can be an important factor in determining cefazolin pharmacokinetics [19], our small sample size did not allow us to use weight as a covariate and future studies are needed to further define optimal cefazolin dosing in this population. Nonetheless, this is the first study to address variability in cefazolin penetration among patients with chronic wound infections. Our results are also consistent with the current recommended dosing regimen for treatment of Enterobacteriaceae based on the CLSI breakpoint (i.e., $2 \mathrm{~g} \mathrm{q} 8 \mathrm{~h}$ ), as well as the dosage typically utilized for $S$. aureus skin and soft tissue infections (i.e., $1 \mathrm{~g}$ q8h).

\section{CONCLUSION}

Cefazolin penetration into the ISF of a lower limb infection varied across this simulated patient population. Cefazolin $1 \mathrm{~g}$ q8h $f \mathrm{~T}>\mathrm{MIC}$ exposures in serum were high at MICs of $1 \mathrm{mg} / \mathrm{L}$ and $2 \mathrm{mg} / \mathrm{L}$. Based on the tissue ISF exposure in this mixed obese and non-obese population with normal renal function, $1 \mathrm{~g}$ q8h dose regimen should be sufficient to achieve 30\% $f \mathrm{~T}>$ MIC target against most MSSA causing cSSTI. However, for Enterobacteriaceae with 
MIC of $2 \mathrm{mg} / \mathrm{L}$, PTAs for the established 50\% $f \mathrm{~T}>$ MIC target were not optimal with $1 \mathrm{~g}$ q8h dose. At least $2 \mathrm{~g}$ q8h dose is required to achieve PTA of $\geq 80 \%$ when treating cSSTI caused by susceptible Enterobacteriaceae (MIC $\leq 2 \mathrm{mg} / \mathrm{L}$ ).

\section{ACKNOWLEDGMENTS}

This work was supported internally by the Center for Anti-Infective Research and Development, Hartford Hospital, Hartford, CT, USA. We acknowledge Amira Bhalodi, PharmD and Seth Housman, PharmD for collection of the original cefazolin serum and tissue concentration data, and Michael Neely, MD for his guidance during the population pharmacokinetic analysis and Monte Carlo simulation. The contents of this article were presented as a poster at the 54th Interscience Conference on Antimicrobial Agents and Chemotherapy in Washington, DC, USA. All named authors meet the ICMJE criteria for authorship for this manuscript, take responsibility for the integrity of the work as a whole, and have given final approval for the version to be published.

Conflict of interest. Wonhee So, Joseph L. Kuti, and David P. Nicolau declare that they have no conflict of interest.

Compliance with ethics guidelines. The analysis in this article is based on a previously conducted study and does not involve any new studies with human or animal subjects performed by any of the authors.

Open Access. This article is distributed under the terms of the Creative Commons Attribution Noncommercial License which permits any noncommercial use, distribution, and reproduction in any medium, provided the original author(s) and the source are credited.

\section{REFERENCES}

1. Zervos MJ, Freeman K, Vo L, et al. Epidemiology and outcomes of complicated skin and soft tissue infections in hospitalized patients. J Clin Microbiol. 2012;50(2):238-45.

2. Garau J, Ostermann H, Medina J, et al. Current management of patients hospitalized with complicated skin and soft tissue infections across Europe (2010-2011): assessment of clinical practice patterns and real-life effectiveness of antibiotics from the REACH study. Clin Microbiol Infect. 2013;19(9):E377-85.

3. Cefazolin package insert. http://www. sagentpharma.com/Products/Cefazolin/Catalog/ Cefazolin_PI1.pdf?PHPSESSID=22da06f6533ba6c35 76d3c28d8b41882. Accessed June 16, 2014.

4. Housman ST, Sutherland CA, Nicolau DP. Pharmacodynamic profile of commonly utilisedparenteral therapies against methicillinsusceptible/resistant Staphylococcus aureus from US hospitals. (Abstract No. eP178). The 24th European Congress of Clinical Microbiology and Infectious Diseases, Barcelona, Spain, May 2014.

5. Paterson DL, Ko WC, Von Gottberg A, et al. Outcome of cephalosporin treatment for serious infections due to apparently susceptible organisms producing extended-spectrum $\beta$-lactamases: implications for the clinical microbiology laboratory. J Clin Microbiol. 2001;39(6):2206-12.

6. Clinical and Laboratory Standards Institute. 7th ed. M100 S20-S23. CLSI, Wayne, PA, USA; 2012.

7. Turnidge JD. On behalf of the subcommittee on antimicrobial susceptibility testing of the Clinical and Laboratory Standards Institute. Cefazolin and Enterobacteriaceae: rationale for revised susceptibility testing breakpoints. Clin Infect Dis. 2011;52(7):917-24.

8. Bhalodi AA, Housman ST, Shepard A, Nugent J, Nicolau DP. Tissue pharmacokinetics of cefazolin in patients with lower limb infections. Antimicrob Agents Chemother. 2013;57(11):5679-83.

9. Stahle L, Arner P, Ungerstedt U. Drug distribution studies with microdialysis III: extracellular concentration of caffeine in adipose tissue in man. Life Sci. 1991;49(24):1853-8. 
10. Lipsky BA, Berendt AR, Cornia PB, et al. 2012 Infectious diseases society of America clinical practice guideline for the diagnosis and treatment of diabetic foot infections. Clin Infect Dis. 2012;54(12):132-73.

11. Chaurasia CS, Muller M, Bashaw ED, et al. AAPSFDA workshop white paper: microdialysis principles, application, and regulatory perspectives. J Clin Pharmacol. 2007;47(5): 589-603.

12. Dudley $\mathrm{MN}$, Nightingale $\mathrm{CH}$, Drezner $\mathrm{AD}$, Low $\mathrm{HB}$, Wuintiliani R. Comparative penetration of cefonicid and cefazolin into the atrial appendage and pericardial fluid of patients undergoing openheart surgery. Antimicrob Agents Chemother. 1984;26(3):347-50.

13. Neely MN, van Guilder MG, Yamada WM, Schumitzky A, Jelliffe RW. Accurate detection of outliers and subpopulations with Pmetrics, a nonparametric and parametric pharmacometric modeling and simulation package for R. Ther Drug Monit. 2012;34(4):467-76.

14. Yamaoka K, Nakagawa T, Uno T. Application of Akaike's information criterion (AIC) in the evaluation of linear pharmacokinetic equations. J Pharmacokinet Biopharm. 1978;6(2):165-75.

15. Cockcroft DW, Gault MH. Prediction of creatinine clearance from serum creatinine. Nephron. 1976;16(1):31-41.

16. Winter MA, Guhr KN, Berg GM. Impact of various body weights and serum creatinine concentrations on the bias and accuracy of the Cockcroft-Gault equation. Pharmacotherapy. 2012;32(7):604-12.

17. van Kralingen S, Taks M, Diepstraten J, et al. Pharmacokinetics and protein binding of cefazolin in morbidly obese patients. Eur J Clin Pharmacol. 2011;67(10):985-92.

18. Douglas A, Udy AA, Wallis SC, et al. Plasma and tissue pharmacokinetics of cefazolin in patients undergoing elective and semi-elective abdominal aortic aneurysm open repair surgery. Antimicrob Agents Chemother. 2011;55(11):5238-42.

19. Brill MJE, Houwink API, Schmidt S, et al. Reduced subcutaneous tissue distribution of cefazolin in morbidly obese versus non-obese patients determined using clinical microdialysis. J Antimicrob Chemother. 2014;69(3):715-23.

20. Muller $M$, dela Pena $A$, Derendorf $H$. Issues in pharmacokinetics and pharmacodynamics of antiinfective agents: distribution in tissue. Antimicrob Agents Chemother. 2004;48(5):1441-53.

21. MacGowan AP, Bowker KE. Continuous infusion of beta-lactam antibiotics. Clin Pharmacokinet. 1998; 35(5):391-402.

22. Craig WA. Pharmacokinetic/pharmacodynamic parameters: rationale for antibacterial dosing of mice and men. Clin Infect Dis. 1998;26(1):1-12.

23. Keel RA, Crandon JL, Nicolau DP. Efficacy of human simulated exposures of ceftaroline administered at 600 milligrams every 12 hours against phenotypically diverse Staphylococcus aureus isolates. Antimicrob Agents Chemother. 2011;55(9):4028-32.

24. Andes D, Craig WA. Pharmacodynamics of a new cephalosporin, PPI-0903 (TAK-599), active against methicillin-resistant Staphylococcus aureus in murine thigh and lung infection models: identification of an in vivo pharmacokineticpharmacodynamic target. Antimicrob Agents Chemother. 2006;50(4):1376-83.

25. Drusano GL. Antimicrobial pharmacodynamics: critical interactions of 'Bug and Drug'. Nat Rev Microbiol. 2004;2(4):289-300.

26. Craig WA, Andes DR. In vivo pharmacodynamics of ceftobiprole against multiple bacterial pathogens in murine thigh and lung infection models. Antimicrob Agents Chemother. 2008;52(10): 3492-6. 